\section{CONCILIACIÓN ESTUDIANTIL- FAMILIAR: UN ESTUDIO CUALITATIVO SOBRE LAS LIMITACIONES QUE AFECTAN A LAS MADRES UNIVERSITARIAS}

\author{
Inés Lozano Cabezas \\ Universidad de Alicante \\ ines.lozano@ua.es \\ Marcos Jesús Iglesias Martínez \\ Universidad de Alicante \\ marcos.iglesias@ua.es \\ Amparo Alonso-Sanz \\ Universitat de València \\ M.Amparo.Alonso@uv.es
}

Cómo citar este artículo/Citation: Lozano Cabezas, I., Iglesias Martínez, M. J. y Alonso-Sanz, A. (2016). Conciliación estudiantil-familiar: un estudio cualitativo sobre las limitaciones que afectan a las madres universitarias. Arbor, 192 (780): a341. doi: http://dx.doi.org/10.3989/arbor.2016.780n4017

Recibido: 24 febrero 2015. Aceptado: 10 mayo 2015.

RESUMEN: La conciliación estudiantil-familiar, considerada como la oportunidad de compaginar la maternidad con una vida académica, todavía hoy no se contempla con normalidad en la Educación Superior, lo que genera una discriminación, sobre todo, en las madres universitarias con relación a sus compañeros. A través de una entrevista semiestructurada, y adoptando una metodología cualitativa basada en narrativas, en este estudio, analizamos los pensamientos y las actitudes de un total de 73 universitarias/os, que reflexionan sobre los aspectos de la carrera académica que restringen la conciliación estudiantil-familiar. Los resultados nos ofrecen las limitaciones académicas que según el alumnado dificultan las posibilidades de tener hijas/os, sus propuestas legislativas de mejora y sus actitudes al respecto. Concluimos con la necesidad de actuaciones legislativas concretas y campañas de sensibilización, para la comunidad universitaria y la sociedad en general, que garanticen la igualdad entre mujeres y hombres en el contexto de la Educación Superior.

PALABRAS CLAVE: género; educación superior; conciliación estudiantil-familiar; madres universitarias; narrativas.

\section{FAMILY-STUDENT BALANCE: A QUALITATIVE STUDY ABOUT LIMITATIONS OF UNIVERSITY MOTHERS}

Copyright: (c) 2016 CSIC. Este es un artículo de acceso abierto distribuido bajo los términos de la licencia Creative Commons Attribution (CC BY) España 3.0.

ABSTRACT: The balance between family and student life, viewed as the possibility of being a mother and university student at the same time, is still not seen as something usual in higher education. This leads to discrimination against student mothers compared with other students. In this study, using a qualitative, narrative-based methodology based on semi-structured interviews, we analysed the beliefs and attitudes of a total of 73 university students. They reflect on aspects of the academic career that restrict the student-family balance. The students' opinions revealed the limitations on the possibilities of having children, included some proposals for improvements to the legislation, and their attitudes to the issue. We conclude that there is a need for concrete legislative actions and awareness campaigns addressing both the academic community and the general public, to ensure equality between women and men in higher education.

KEYWORDS: gender; higher education; student-family balance; university mothers; narratives. 


\section{INTRODUCCIÓN}

Para ubicar los márgenes en los que se sitúa este estudio, contextualizado en el ámbito universitario, precisamos una breve descripción de antecedentes cercanos. Las teorías feministas de los años noventa, consiguen el reconocimiento de las mujeres en condiciones de igualdad para que se avance en la consecución de la emancipación racional del ser humano (Amorós, 1987; Amorós, 1990). Además, se constata que la igualdad conseguida es insuficiente y que a pesar de las emergentes políticas hacia la equidad no se demuestra una plena igualdad de género (Camps, 1996). A finales del siglo XX, el énfasis reside en desechar que el empleo femenino sea el matrimonio y los embarazos, mediante el cumplimiento de las normas y leyes que incrementan la posibilidad de la mejora de la igualdad en un estado del bienestar (Valcárcel, 1997). Las mujeres del siglo XXI, emancipadas racionalmente, demuestran que formándose académicamente luchan por su desarrollo personal y profesional a pesar de las condiciones de desigualdad latentes en la institución universitaria. En el sistema educativo español las generaciones actuales tienen oportunidades para seguir formándose a lo largo de la vida, pero la educación aún sigue presentando barreras invisibles que frenan la igualdad en la formación de las mujeres (González Pérez, 2008).

La Universidad es la institución que ha generado la teoría feminista, y por tanto, le corresponde combatir las desigualdades entre mujeres y hombres, creando organismos y políticas que fomenten la equidad. Sin embargo, se detecta una realidad universitaria en la que se produce una clara situación de desigualdad entre alumnas y alumnos, en el momento en que las estudiantes se enfrentan a la conciliación entre su proceso de maternidad y sus estudios.

Actualmente, las conductas universitarias manifiestan un incremento en las edades de acceso a la formación superior. Según datos del Ministerio de Educación, Cultura y Deporte respecto a la población universitaria (Datos básicos del sistema universitario español. Curso 2013-2014), la edad teórica de acceso a la universidad (en torno a los 18-19 años) se está modificando con el reingreso en el sistema de mayores de 30 años. De este modo, la población universitaria responde a diversos perfiles: por un lado, personas en paro que invierten su tiempo en iniciar una formación académica; egresados que deciden continuar la formación universitaria para mejorar sus posibilidades de inserción o reinserción laboral; alumnado que se ve obligado a trabajar para contribuir a la econo- mía familiar; estudiantes con compromisos familiares (maternidad y paternidad), entre otros. Como consecuencia de todo ello, nos encontramos con un colectivo estudiantil diverso y con una amplia dispersión de edad. El aprendizaje a lo largo de la vida, promovido por el Espacio Europeo de Educación Superior (EEES) desde su implantación en el curso 2008/2009 (Real Decreto 1393/2007), conlleva una población más heterogénea y consecuentemente un giro en las necesidades sociales, frente a las que la institución universitaria debe adaptarse y establecer mecanismos para entender el acceso a la universidad como un binomio "derecho a la educación-igualdad de oportunidades", aspecto que han analizado recientemente Lorenzo, Argos, Hernández y Vera (2014, p. 22).

El problema de la desigualdad de género es estructural, procede de muchos ámbitos y afecta a muchas facetas de la mujer. Nos interesa aquí concretamente el ámbito universitario, observamos en la figura 1, que se produce un claro descenso de matrículas de mujeres en estudios de postgrado y más si cabe en las tesis doctorales; mientras que de manera inversa aumenta el número de matrículas de hombres.

Una hipótesis sobre las causas que genera el descenso de matrículas de mujeres en estudios posteriores al grado, es la proximidad a una edad en la que se produce disminución de la fertilidad, junto con otros problemas de desigualdad de género estructurales. Pareciera que la mujer autolimitara su maternidad, retrasando la edad de gestación tanto como le resulta posible hasta superar al menos los estudios universitarios de grado, pero que ante la llegada de una edad en la que posiblemente disminuya su fertilidad, se ve abocada a autolimitarse académicamente. En el contexto español parece más apropiado referirse a la maternidad como posible causa de autolimitación académica, más que únicamente como motivo de deserción estudiantil. $Y$ al igual que en el caso de la predisposición de las estudiantes universitarias a autolimitarse profesionalmente en el futuro por razones de conciliación, entendemos que "las tres explicaciones de las desigualdades de género (capacitación, discriminación y autolimitación) se encuentran interrelacionadas" (López-Ibor, Escot y Fernández, 2010, p. 2). La autolimitación académica de las mujeres, en el contexto de una sociedad avanzada como España, supone ralentizar o renunciar a la progresión académica ante las dificultades o limitaciones para compatibilizar la vida estudiantil con la maternidad y/o el cuidado de sus hijas/os. La autolimitación a la maternidad, en este mismo contexto social, supone ralentizar o re- 
Figura 1. Distribución porcentual del alumnado según el género en las universidades españolas durante el curso académico 2010-2011

\section{Curso académico 2010-2011}

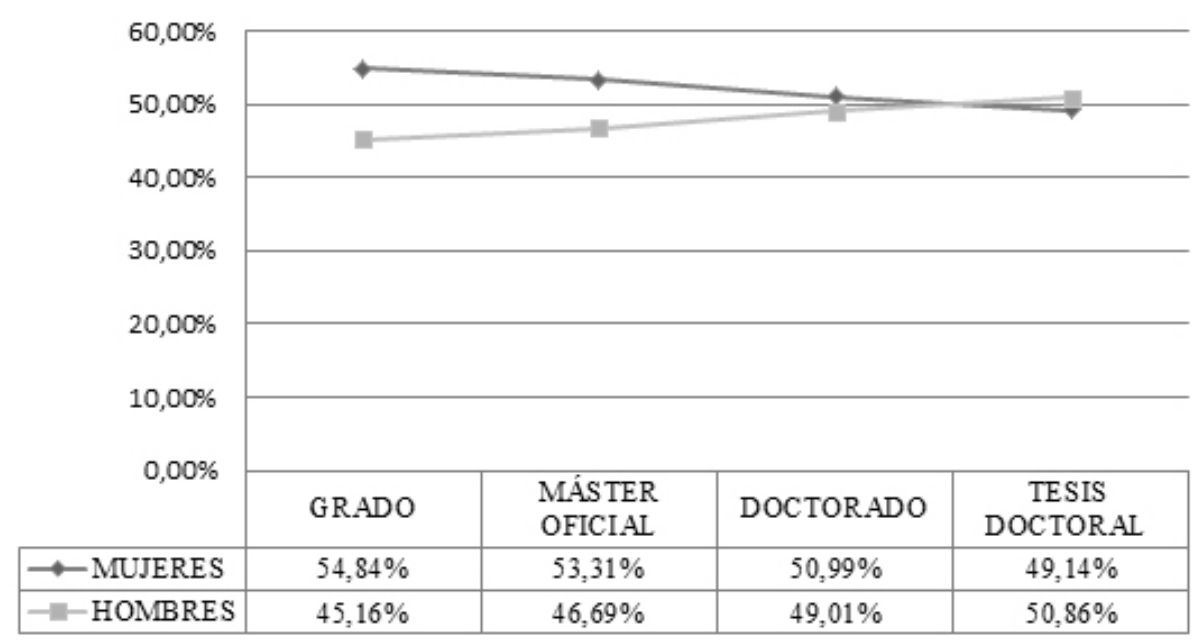

Fuente: Elaboración propia a partir de los datos INEbase: Operaciones estadísticas: clasificación por temas.

nunciar a la gestación o al cuidado de las/os hijas/os ante las dificultades o limitaciones para compatibilizar la vida estudiantil con la maternidad y/o el cuidado de sus hijas/os. Estos obstáculos afectan en mayor medida a las alumnas que desean ser madres que a los alumnos que desean ser padres, porque la gestación es una cuestión de sexo, y al menos durante el periodo de embarazo, puerperio y lactancia natural, las mujeres estudiantes encuentran algunas limitaciones que no encuentran los hombres.

Asimismo, las investigadoras Donoso, Figuera y Rodríguez-Moreno (2011) hallan evidencias de los factores que pueden dificultar el proyecto profesional de las estudiantes universitarias. De las percepciones del rol de género en el mundo laboral determinan su proceso de categorización "rasgos estereotipados femeninos", en el que el esfuerzo de compatibilizar la familia con el trabajo tiene mayor relevancia e implicación en el caso de las mujeres que en el de los hombres. Estudios relativos a la corresponsabilidad familiar para el logro de la conciliación familiar (Maganto, Extxebería y Porcel, 2010), exponen que las mujeres pueden sufrir una sobrecarga de roles; sin embargo, consideramos que este hecho no solo es experimentado por las mujeres trabajadoras, sino también, por aquellas estudiantes que concilian su estudios con la maternidad. Por tanto, estas cuestiones relativas al rol enfatizan que los obstáculos de la autolimitación académica y a la maternidad afecten en mayor medida a las alumnas.

\section{La maternidad durante los periodos de estudios universitarios}

Saletti (2008) realiza una revisión bibliográfica teórico-empírica sobre el concepto de maternidad en las teorías feministas. El análisis de estos discursos feministas ayudan a cuestionar los mandatos sociales que recaen sobre la maternidad y las mujeres, y esta reflexión permite plantear la posibilidad de cambios sociales y políticos hacia la igualdad entre mujeres y hombres. En este sentido, la citada autora desglosa en su revisión dos bloques en los cuales se enclavan las teorías feministas: el primero sitúa a la maternidad en la "deconstrucción del instinto maternal", y el segundo como fuente de placer exclusivamente femenino.

Según Saletti (2008) la primera postura se fundamenta en la teoría de Simone De Beauvoir que considera la maternidad como un deseo femenino ambivalente que no incluye ni lo maternal ni lo antimaternal, sino que es un hecho social que anula a 
la mujer como persona, pues las/os hijas/os son un obstáculo para el desarrollo de las mujeres en la sociedad. "Fue la primera en desmitificar el "instinto maternal" e interpretarlo como instrumento de dominación" (Simone de Beauvoir, 1949, p. 41; citado por Burgaleta, 2011). En 1981 Elizabeth Badinter retoma esta tesis para desmontar los mitos desarrollados en el siglo XIX adscritos al "instinto maternal", prejuicios aceptados comúnmente respecto a la idea de la realización plena de la mujer a través de la experiencia de ser madre, "para inducir a las mujeres a consagrarse al cuidado de sus hijos" (p. 42, citado por Burgaleta, 2011).

La segunda postura se corresponde con aquellas teorías feministas que proponen la maternidad como fuente de placer, conocimiento y poder intrínseco y exclusivamente femenino, evitando, por tanto, que las madres se resignen a ser víctimas de la cultura patriarcal o institucional.

El análisis de los discursos feministas en torno a la maternidad, en el contexto actual nos permite extraer que las mujeres para su realización plena, no precisan consagrarse en exclusividad al cuidado de sus hijas/ os y muchas, tampoco necesitan ser madres. Sin embargo, aquellas mujeres que desean ser madres y que para su autorrealización optan por desarrollar una carrera académica, sí requieren de instituciones que garanticen la conciliación estudiantil-familiar para no sostener la maternidad como instrumento de dominación de la cultura patriarcal. Nosotras definimos la conciliación estudiantil-familiar como el equilibrio entre el cumplimiento de las obligaciones estudiantiles y las responsabilidades familiares, posible únicamente cuando las condiciones académicas, legislativas y relativas a recursos lo garantizan.

Las campañas de sanidad iniciadas en los 90, para la prevención de las enfermedades de trasmisión sexual, provocan una disminución de los embarazos no deseados; de manera que a inicios del siglo XXI, la maternidad en España no se contempla como un problema que ocasione abandono de la formación superior. Así, se comprueba mediante los datos de la Encuesta de fecundidad 1999 del Instituto Nacional de Estadística, en los que la maternidad es considerada como causa del abandono de los estudios solamente en un $2 \%$ de las mujeres.

Comienza un periodo en el que la mayoría de las mujeres españolas cuentan con suficientes medidas preventivas que les permiten quedar embarazadas cuando así lo desean. En el Instituto Nacional de
Estadística no existen datos, posteriores al 1999 y relativos a la maternidad en mujeres estudiantes, que podamos consultar. Sin embargo, unos años más tarde, según datos del Centro de Investigaciones Sociológicas (2006), en el Estudio no 2.639 sobre Fecundidad y valores en la España del siglo XXI, distribuciones marginales de la Comunidad Valenciana, de la pregunta $406^{1}$ se trasluce que, entre las mujeres que tienen menos de 50 años y están embarazadas en el momento de la entrevista, el 94,1\% desea el embarazo. Durante este periodo, desde el año 2000 hasta el 2006, la confluencia entre compromisos estudiantiles y familiares deviene solamente en causa de autolimitación académica; tal y como lo demuestra la literatura revisada (Cabrera, Bethencourt, Álvarez y González, 2006; Escandell, Marrero, Castro y Rodríguez, 2002) que no refiere las cargas familiares como una de las posibles causas de abandono de los estudios universitarios.

Sin embargo, las cargas familiares (Andreu, 2008) y su incompatibilidad con las actividades académicas (Elboj Saso, Iñiguez Berrozpe y Valero Errazu, 2013) se consideran de nuevo causa de abandono de los estudios universitarios a partir de la crisis económica en el año 2007. El inicio de la crisis altera el perfil universitario con el reingreso en el sistema de mayores de 30 años y, por tanto, de más mujeres madres o en vías de serlo. De manera que en la actualidad la maternidad, en el contexto universitario español, además de ser causa de autolimitación académica se considera de nuevo como una de las posibles causas de abandono de los estudios. Con independencia de que los embarazos sean voluntarios o involuntarios, la maternidad no debe ser en ningún caso un problema que obligue a las mujeres a abandonar su carrera académica, pues supone una clara situación de discriminación por razón de sexo.

Las mujeres españolas estudiantes, en general, suelen esperar a tener una situación de emancipación, desarrollo académico, profesional, laboral, económico y familiar, adecuados para tener descendencia, y en esta línea las universitarias retrasan la concepción hasta finalizar, como mínimo, los estudios de grado. Pero también nos encontramos en las aulas con estudiantes embarazadas y con estudiantes que ya eran madres antes de acceder a la Universidad, aunque sean una minoría. La mujer que se enfrenta a la decisión de desarrollarse académicamente y/o ser madre debe lidiar con actitudes contrarias a la conciliación estudiantil-familiar. Estas creencias se transmiten a las mujeres a través de influyentes mensajes 
enclavados social y culturalmente, como consecuencia de la falta de estructuras y políticas de justicia social que garanticen un apoyo a la mujer estudiante. Si la sociedad dotara de apoyos académicos, legales y económicos, en favor de la conciliación estudiantilfamiliar, a las mujeres estudiantes; estas no se verían abocadas a autolimitar la maternidad a causa de dificultades y obstáculos, sino que decidirían de forma totalmente voluntaria y no condicionada sobre la posibilidad de ser madres. Incluso las mujeres madres podrían plantearse con normalidad la alternativa de continuar, de ingresar o reingresar en el sistema académico universitario para incrementar su formación durante cualquier periodo: de desempleo, de bajas por maternidad, de excedencias laborales, mientras trabajan... Estas medidas podrían reducir tanto la autolimitación a la maternidad como la autolimitación académica de las mujeres.

\section{Mecanismos de las instituciones universitarias para la conciliación estudiantil-familiar}

La implantación del EEES trae consigo cambios pedagógicos, como la práctica de metodologías de enseñanza-aprendizaje más activas y centradas en el estudiante (aprendizaje basado en proyectos, trabajo colaborativo); cambios en los procesos evaluativos, con mayor peso de la evaluación continua, períodos de prácticas y obligatoriedad de asistencia a clase; y cambios en las nomenclaturas, como los créditos ECTS con los que se programa la carga lectiva dentro y fuera de las aulas. Si bien estos cambios pueden implicar al alumnado en sus estudios también pueden perjudicar a quienes tratan de compatibilizar sus estudios con una actividad laboral o con cargas familiares (Elboj Saso, Iñiguez Berrozpe y Valero Errazu, 2013).

El problema de la incompatibilidad estudiantillaboral puede afectar igualmente a hombres y mujeres. Sin embargo, tal y como hemos manifestado anteriormente, la incompatibilidad entre estudios y maternidad/paternidad afecta en mayor medida a las mujeres debido a que los procesos biológicos los experimentan solamente ellas. Frente a esta problemática, de incompatibilidad estudiantil-familiar en el contexto universitario, existen múltiples respuestas: disminución del tiempo dedicado al cuidado y educación de las/os hijas/os, imposibilidad de respetar el periodo de baja maternal si se desea dar continuidad a los estudios, obstáculos para lactar al recién nacido, dificultades para afrontar económicamente los gastos derivados de la maternidad, así como la frustración e insatisfacción por la autolimitación de la maternidad.
Esto genera una condición de desigualdad y discriminación hacia la mujer, que la institución universitaria no debe permitir.

Los mecanismos que poseen las instituciones universitarias en favor de la conciliación estudiantil-familiar no son completamente efectivos. Las Leyes Orgánicas $1 / 2004,3 / 2007$ y 4/2007, promueven la creación de unidades u observatorios de igualdad en las Universidades españolas mediante el compromiso de desarroIlar las funciones relacionadas con el principio de igualdad entre mujeres y hombres. Sin embargo, los Planes de Igualdad no son un proceso uniforme y común característico de todas las Universidades, sino que tienen identidad propia referida a la institución universitaria en la que se desarrolla (Cerdá, 2009).

En suma, los Planes de Igualdad delimitan una serie de acciones orientadas a: establecer un plan estratégico de la institución universitaria; realizar un análisis previo de la situación de cada una de las Universidades, referidas a los datos socio-demográficos de la comunidad universitaria (alumnas/os, personal de administración y servicios y personal docente e investigador); conocer y detectar las discriminaciones horizontales y verticales en lo que se refiere a los cargos de gestión; y, finalmente, plantear la propuesta y la posterior aplicación en los distintos ámbitos de actuación en los que han de implicarse todos los miembros de la comunidad universitaria. En general, los ámbitos a los cuáles se dirigen las propuestas de los Planes de Igualdad universitarios son dirigidos a: política de igualdad de oportunidades; responsabilidad con la sociedad; comunicación, imagen y lenguaje institucional; representatividad de las mujeres en los cargos de gestión y gobierno; acceso, selección, promoción y desarrollo; conciliación de la vida familiar, laboral y personal; políticas feministas en contra del acoso, actitudes sexistas y percepción de discriminación; así como, condiciones laborales y condiciones físicas en el entorno de trabajo. Estos Planes están siendo un gran avance para la consecución de la conciliación laboral-familiar entre las académicas (Tomàs Folch y Mentado Labao, 2013), pero no han respondido a las demandas de las madres estudiantes.

Por otro lado el Estatuto del Estudiante Universitario (Real Decreto 1791/2010) establece para el alumnado, el derecho a "una atención y diseño de las actividades académicas que faciliten la conciliación de los estudios con la vida laboral y familiar" (p. 109357) pero no existen regulaciones más concretas que garanticen su cumplimiento. También establece para los estudiantes de Grado y Máster, el derecho específico "a elegir grupo de 
docencia, en su caso, en los términos que disponga la universidad, de forma que se pueda conciliar la formación con otras actividades profesionales, extra-académicas o familiares" (p. 109359). Este es el único artículo del Real Decreto 1791/2010, enfocado a garantizar la conciliación; pero no es suficiente, pues se trata de un artículo ambiguo, poco específico y nada determinante.

\section{METODOLOGÍA}

Los resultados de este artículo forman parte de una amplia investigación plurinstitucional (Universidad de Alicante y Universitat de València) que abarca un problema complejo como el de la conciliación estudiantilfamiliar analizado desde distintas perspectivas. De las cuales en este caso interesan las relacionadas particularmente con un enfoque centrado en la maternidad de las estudiantes, cuyos objetivos son:

- Conocer y analizar las dificultades o limitaciones que la carrera académica imprime en la maternidad del colectivo estudiantil universitario, debido a la falta de conciliación estudiantil-familiar.

- Identificar posibles soluciones o propuestas legislativas de mejora, desde el punto de vista del estudiante universitario, que favorezcan el derecho a la maternidad o paternidad del colectivo estudiantil.

- Analizar y describir las actitudes de los estudiantes en favor a la conciliación estudiantilfamiliar en el contexto universitario.

El contexto de este estudio se enmarca desde la realidad de que en los últimos años se ha producido un aumento de estudiantes en estado de gestación durante sus estudios universitarios, y también estudiantes que acceden a los mismos siendo ya madres o padres. Las madres universitarias además no se haIlan solamente en estudios de postgrado (Programas de Doctorado o Estudios Oficiales de Postgrado), sino también empiezan a ser una realidad en los estudios de Grado. Concretamente en el Grado de Maestra/o, en el que desarrollamos esta investigación, por tratarse de estudios con mayor presencia de mujeres que de hombres (Tabla I), los casos de alumnas embarazadas pueden ser más frecuentes.

La sensibilidad hacia temas relacionados con procesos biológicos femeninos como el embarazo, el parto y posparto, y su incompatibilidad con los estudios; se espera que sea mayor en aquellas titulaciones con mayor presencia de mujeres. Aunque el tema resulte de interés para mujeres y hombres, la necesidad parte en realidad enfocada hacia una población femenina. Es por ello que nos parece interesante comenzar este estudio
Tabla I. Número de alumnado matriculado en la el Grado de Maestro (Universidad de Alicante y Universitat de València)

\begin{tabular}{lccc}
\hline & Mujeres & Hombres & Total \\
\hline UA & 563 & 152 & 715 \\
\hline UV & 2559 & 663 & 3222 \\
\hline
\end{tabular}

Datos facilitados por la Unidad Técnica de Calidad (UTC) de la Universidad de Alicante ${ }^{2}$.

Datos facilitados por el Servei d’Anàlisi i Planificació (SAP) de la Universitat de València.

con la población del Grado de Maestro en Educación. Concretamente se invita a participar a 116 estudiantes pertenecientes al Grado de Maestro de Educación Primaria de la Universidad de Alicante (UA) y de la Universitat de València (UV). La muestra la componen finalmente un total de 73 personas, de ellas son, $76,72 \%$ mujeres, frente a un $23,29 \%$ de hombres.

Tal y como se observa en la figura 2, la mayoría de los estudiantes de Grado tienen una edad comprendida mayoritariamente entre los 18 y los 25 años; sin embargo, las modalidades de ingreso a la universidad a través de la formación profesional, mayores de 25 años y otras opciones se encuentran al alza. Estos datos nos hablan de un crecimiento de la población adulta en las universidades de Alicante y Valencia, a la que se le presupone mayor madurez en la toma de decisiones, en una franja de edad que se acerca al límite de la edad fértil y que, posiblemente, se plantea tener descendencia a la vez que estudia o decida descartar esta posibilidad por incompatibilidad estudiantil-familiar.

Figura 2. Porcentajes de edades de los participantes

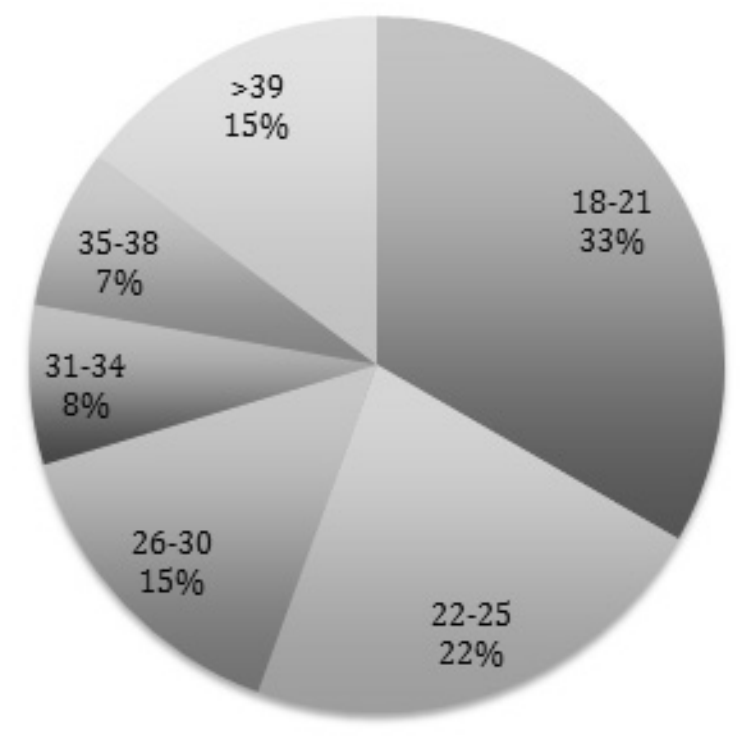


Desde una perspectiva plurinstitucional (Universidad de Alicante y Universitat de València) se comparan las opiniones del alumnado, respecto a la conciliación estudiantil-familiar. Para ello se eligen 2 grupos de población de rasgos similares y diferentes instituciones, de manera que se evita la contaminación entre ellos. Con el grupo control de 27 estudiantes de la Facultat de Magisteri de la Universitat de València (UV), se lleva a cabo un proceso de sensibilización mediante un grupo de discusión sobre la temática, que finaliza con una performance que conceptualiza la idea de la maternidad/paternidad en el contexto universitario. Mientras que en el grupo de 46 participantes de la Facultad de Educación de la Universidad de Alicante (UA), no se realiza ninguna campaña de sensibilización o reflexión previa a la investigación.

Para la recogida de datos optamos por una entrevista semiestructurada de respuesta abierta que se facilita al alumnado, por ser uno de los procedimientos más frecuentes utilizados en los estudios de carácter cualitativo (Denzin y Lincoln, 2012), ya que permite que los participantes profundicen en sus pensamientos y experiencias (Clandinin y Murphy, 2009) durante su vida académica (Huber, Caine, Hubery Steeves, 2013). La entrevista plantea la reflexión frente a tres cuestiones sobre la problemática del estudio:

- Aspectos de la carrera académica que limitan las posibilidades de tener hijas/os.

- Propuestas legislativas de mejora de la conciliación estudiantil-familiar.

- Actitudes del alumnado adoptables a favor de la conciliación estudiantil-familiar.

Esta investigación se enmarca dentro del paradigma feminista de corte cualitativo, por formar parte de la discusión emergente de nuevas oportunidades en el análisis de la justicia social (Olesen, 2011), ya que analiza e interpreta la variedad de relatos reflexivos de las participantes. En base a estas se elabora el boceto del mapa de códigos, que tras una lectura iterativa de los relatos se reelabora, para realizar una revisión y redefinición de los códigos. Posteriormente, este sistema de codificación se valida desde una perspectiva interdisciplinar, por dos expertos del área de Didáctica y Organización Escolar y una experta del área de Didáctica de la Expresión Plástica. Finalmente, y en base a la configuración definitiva del mapa de códigos, se procede al análisis de las narrativas (con razonamiento tanto inductivo como deductivo), respetando en todo momento las prescripciones propias de la metodología cualitativa (Corbin y Strauss, 2008), articulando los códigos emergentes de los relatos con la estructura científica del marco conceptual para lograr una comprensión más profunda del fenómeno de estudio del paradigma feminista en la investigación cualitativa (Olesen, 2011).

El tratamiento de los datos se realiza mediante el programa de análisis cualitativo (Analysis Qualitative Data) AQUAD 7 (Huber y Gürtler, 2013) herramienta que conjuga el proceso de interpretación y de codificación, con la emergencia de categorías provenientes de las propias voces de los participantes. Esta investigación se centra en una perspectiva paradigmática cualitativa, que busca garantizar la fiabilidad y validez del estudio, a través de procedimientos informáticos de tratamiento de datos que ha sido proporcionado por el citado programa. Además se completa el proceso cualitativo con una cuantificación a través del porcentaje de la frecuencia absoluta (\%FA) de los códigos determinados, así como la frecuencia relativa (FR) que corresponde al valor de la frecuencia absoluta partido por la muestra. Para garantizar el anonimato de los participantes, cada cuestionario se identifica con un código alfanumérico: esuamp000 (Estudiante Universidad de Alicante, Educación Primaria, Género y el número del cuestionario) y esuvhp000 (Estudiante Universitat de València, Educación Primaria, Género y número del cuestionario).

\section{RESULTADOS}

Finalizado el proceso de codificación de las unidades de narrativa y en base a la constitución del mapa de códigos han emergido un total de tres categorías: 1. Aspectos de la carrera académica que limitan las posibilidades de tener hijas/os; 2. Propuestas legislativas de mejora de la conciliación estudiantil-familiar; y 3. Actitudes del alumnado adoptables a favor de la conciliación estudiantil-familiar. Para la exposición de los resultados se utilizan tablas que agrupan los códigos en cada una de las categorías con sus diferentes frecuencias de aparición (\%FA), diferenciando en columnas las dos instituciones participantes.

\section{Categoría 1. Aspectos de la carrera académica que limitan las posibilidades de tener hijas/os}

Las reflexiones de los estudiantes han permitido determinar algunas limitaciones u obstáculos que dificultan la posibilidad de tener hijos/as. Del análisis de las narrativas han emergido un total de 9 códigos los cuales han sido aglutinados en la categoría 1 y se presentan en la tabla II. 
Tabla II. Frecuencias absolutas de la categoría 1

\begin{tabular}{lcccc}
\hline CóDIGOS & \%FA UV & FR UV & \%FA UA & FR UA \\
\hline 1.1 Carga de trabajo académico & $31,58 \%$ & 0,44 & $58,11 \%$ & 0,93 \\
\hline 1.2 Gasto económico derivado de los estudios & $18,42 \%$ & 0,26 & $21,62 \%$ & 0,35 \\
\hline 1.3 Duración de la carrera universitaria & $2,63 \%$ & 0,04 & $1,35 \%$ & 0,02 \\
\hline 1.4 Horarios establecidos & $21,05 \%$ & 0,30 & $1,35 \%$ & 0,02 \\
\hline 1.5 Sistema evaluación & $7,89 \%$ & 0,11 & $1,35 \%$ & 0,02 \\
\hline 1.6 Relación social & $2,63 \%$ & 0,04 & $0,00 \%$ & 0,00 \\
\hline 1.7 Exigencias físicas & $2,63 \%$ & 0,04 & $2,70 \%$ & 0,04 \\
\hline 1.8 Prioridad estudios & $10,53 \%$ & 0,15 & $12,16 \%$ & 0,20 \\
\hline 1.9 Sin limitaciones & $2,63 \%$ & 0,04 & $1,35 \%$ & 0,02 \\
\hline TOTAL & $100 \%$ & - & $100 \%$ & - \\
\hline
\end{tabular}

Las narrativas con una frecuencia más alta son las referidas a la carga de trabajo que supone estudiar y que es difícil de compaginar con la maternidad (código 1.1 Carga de trabajo académico) y los factores económicos (código 1.2 Gasto económico derivado de los estudios). El tiempo requerido para estudiar, y el gasto que supone costearse los materiales precisos (fotocopias, libros, equipamiento, fungibles...) así como el pago de las matrículas cada vez más elevado $y$, especialmente, cuando no se aprueba en la primera convocatoria (aspecto probable en caso de coincidencia de embarazo o puerperio durante el curso): "ya que con el dinero gastado en los estudios se dificulta el poder mantener hijos tan pequeños mientras se cursan estos" (esuamp056).

Los horarios de asistencia a clase (código 1.4 Horarios establecidos) es otra de las razones aducidas, ya que obliga al alumnado universitario a optar por un turno fijo, y "sin opción a poder escoger horarios en asignaturas de mañanas y tarde" (esuvmp09), lo cual dificulta la compatibilidad de asistencia a clase y el cuidado de la descendencia fuera del horario escolar. Además la elección de horario se realiza dando prioridad a los mejores expedientes académicos y sin contemplar una posible prioridad de elección a las madres o padres.

El sistema de evaluación que impera en la actualidad en las universidades españolas (código 1.5 Sistema evaluación), se caracteriza por un "complicado horario presencial en la Universidad, y la cantidad de trabajos" (esuvmp070) a los que el alumnado debe responder en continuos entregables y no durante un concreto y breve periodo de exámenes, como ocurría antes de la implantación del EEES. Además en la mayoría de las ocasiones se evalúa el resultado de un proceso colaborativo (código 1.6 Relación social) que implica para el estudiante "cantidad de trabajos, especialmente grupales para los que debes reunirte y estar disponible en casi todo momento" (esuvmp070).

Se considera la duración de los estudios (código 1.3 Duración de la carrera universitaria) como otro de los factores limitantes por interferir en el período de edad fértil. Asimismo, el alumnado recuerda además algunas problemáticas que únicamente afectan a las mujeres estudiantes (código 1.7 Exigencias físicas): “depende de la carrera que se esté realizando, por ejemplo, en Ciencias de la Actividad Física y el Deporte, no se podría realizar la parte práctica" (esuamp006). Refiriéndose a momentos en los que cualquier médico no recomendaría esfuerzos físicos, como el puerperio, un avanzado estado de gestación o un embarazo de riesgo. Por otra parte, prevalece el desarrollo académico que permita una estabilidad económica y profesional antes de la con la que afrontar la maternidad (código 1.8 Prioridad estudios): "los estudiantes no suelen plantearse tener hijos en esta etapa de su vida, deben formarse para en un futuro tener un nivel de vida que les permita mantener y cuidar a sus hijos" (esuvhp021). Y aunque una pequeña minoría cree que no existen limitaciones a la maternidad/paternidad (código 1.9 Sin limitaciones), "que la carrera no impide tener hijos" (esuamp064); esta mirada se vuelve más optimista en el grupo de la Universidad de Valencia en el que ha habido un proceso de sensibilización hacia la compatibilidad estudiantil-familiar.

\section{Categoría 2. Propuestas legislativas de mejora para la conciliación estudiantil-familiar}

Los resultados referidos a las propuestas legislativas que plantean los estudiantes para la mejora de la conciliación estudiantil-familiar han sido agrupados en la categoría 2 y mostrados en la tabla III. 
Tabla III. Frecuencias absolutas de la categoría 2

\begin{tabular}{|c|c|c|c|c|c|}
\hline CóDIGOS & SUBCÓDIGOS & $\%$ FA UV & FR UV & \%FA UA & FR UA \\
\hline \multicolumn{2}{|c|}{ 2.1 Baja maternidad estudiantil } & $14,29 \%$ & 0,19 & $1,33 \%$ & 0,02 \\
\hline \multirow{4}{*}{ 2.2 Ayudas económicas } & 2.2.1 Becas de estudios & $28,57 \%$ & 0,37 & $57,33 \%$ & 0,93 \\
\hline & 2.2.2 Becas de transporte & $5,71 \%$ & 0,07 & $9,33 \%$ & 0,15 \\
\hline & 2.2.3 Reducción tasas matrícula & $20,00 \%$ & 0,26 & $25,33 \%$ & 0,41 \\
\hline & 2.2.4 Fragment. pago de tasas & $0,00 \%$ & 0,00 & $6,67 \%$ & 0,11 \\
\hline \multicolumn{2}{|l|}{ 2.3 Asistencia con bebé } & $28,57 \%$ & 0,37 & $0,00 \%$ & 0,00 \\
\hline \multicolumn{2}{|c|}{ 2.4 Seguro escolar hijas/os } & $2,86 \%$ & 0,04 & $0,00 \%$ & 0,00 \\
\hline \multicolumn{2}{|l|}{ Total } & $100 \%$ & - & $100 \%$ & - \\
\hline
\end{tabular}

Muchos de estos cambios necesarios en favor de la conciliación estudiantil-familiar se pueden contemplar dentro de las aulas para facilitar el proceso de enseñanza-aprendizaje, hablamos de metodologías y sistemas de evaluación adaptados, uso de recursos on-line, actitudes favorecedoras de alumnado y profesorado, etc. Pero para que los estudiantes puedan llevar a cabo la conciliación estudiantil-familiar en su totalidad necesitamos también cambios a nivel legislativo que apoyen esta demanda.

Casi la totalidad del alumnado de la UA y más de la mitad del alumnado de la UV, requiere ayudas o becas (código 2.2 Ayudas económicas) para poder llevar a cabo esta conciliación. Estas ayudas se demandan de forma general pero también especificando varios tipos (que aquí se analizan como subcódigos), así según la diversidad de testimonios por ejemplo recogemos (subcódigo 2.2.1 Becas de estudios) que "estaría bien que dieran becas a los estudiantes con hijos para pagar guarderías o personas que cuiden a sus hijos mientras ellos asisten a clase" (esuahp038); también "facilitar material que se necesite en el aula" (esuamp050); o entregar a madres y padres "bonos para hacer fotocopias" (esuamp055); pero incluso se contempla la "posibilidad de recibir beca aunque no se matricule en 60 créditos" (esuamp019) -requisito mínimo actual para poder solicitar la beca de estudios-. Y se insiste, especialmente, (subcódigo 2.2.3 Reducción tasas matrícula) en que "quizás facilitaría algo bajar los precios de las matrículas, ya que estudiar y mantener una familia es complicado" (esuamp068), en "por lo menos devolver el dinero de los créditos en los que la alumna no se ha podido presentar" (esuvmp048) o bien "no hacer pagar el doble de tasas por aquellas asignaturas que no has podido cursar mientras estás en un período de baja por maternidad" (esuvmp10), "como último caso si no se pudiera continuar estudiando, que no suponga perder el año y al siguiente matricularte pagando como segunda matrícula" (esuvmp049). El pago fraccionado de las tasas académicas (subcódigo 2.2.4 Fragmentación pago de tasas) es otro de los problemas que se plantean: "sería conveniente que se nos permitieran fragmentar las tasas de la matrícula en más de dos veces" (esuamp045). Otras narrativas se refieren a ayudas del transporte (subcódigo 2.2.2 Becas de transporte): "así también becas para el transporte para las madres que vivan lejos, ya que supone un gasto adicional" (esuamp033).

El alumnado de la Universidad de Valencia, más sensibilizado, ha tenido en cuenta además otro tipo de demandas, como considerar el derecho a una "baja maternal de la mujer estudiante" (código 2.1 Baja maternidad estudiantil) -análoga a la baja de la que gozan las mujeres trabajadoras-, "que exista de alguna manera, la posibilidad de una "baja" maternal, para que al menos los 4 primeros meses los puedas dedicar exclusivamente al cuidado del hija/o y poder reincorporarte, sin haber perdido el ritmo de clase" (esuvmp038). Y que frente a situaciones de baja maternal, la estudiante cuente con una situación que le permita no perder las asignaturas en las que se ha matriculado, pudiendo ser algunas de las medidas legislativas favorecedoras:

- El poder asistir con el bebé a las clases durante esos primeros meses de vida (código 2.3 Asistencia con bebé) o dicho con las propias palabras de una alumna: "permitir a los estudiantes ya sean hombres o mujeres conciliar la vida familiar con la estudiantil, es decir, favorecer el asistir a clase con las/os hijas/os o crear una "solución" mientras los padres/madres de esos niños asisten a clase" (esuvmp055).

- Que la madre que opte por asistir a clase, o simplemente a las instalaciones universitarias, con 
su hija/o pueda contratar un complemento al seguro escolar (código 2.4 Seguro escolar hijas/ os) que respalde al recién nacido dentro de las instalaciones universitarias, es decir "que el seguro universitario cubra la posibilidad de poder cuidar de tu hijo o hija aunque te encuentres en sus instalaciones" (esuvmp010). Pudiéndose contratar este complemento tanto en el momento de la matrícula como en cualquier periodo del curso en el que el estudiante se sabe madre o padre (pues las circunstancias y fechas de parto o recepción de adopción no siempre son predecibles con antelación).

\section{Categoría 3. Actitudes del alumnado adoptables a favor de la conciliación estudiantil-familiar}

Una de las claves más importantes en la consecución de la igualdad de oportunidades son las actitudes sociales. Con este propósito una de las cuestiones que se plantea a los estudiantes es conocer el tipo de actitud que mantendría con sus compañeras madres estudiantes. Los resultados se sintetizan en la tabla IV.

A pesar de que parte del alumnado no ha definido la actitud que adoptaría frente a la conciliación estudiantil-familiar (código 3.5 No define actitud), se manifiestan cuatro actitudes diferenciadas: activa, pasiva, positiva y negativa. Mayoritariamente la actitud positiva (código 3.3) con posicionamientos tales como "pienso que la educación debe ir ligada a las familias y a todo tipo de circunstancias que se presenten" (esuamp061), así como la actitud activa (código 3.1) con afirmaciones tales como la de adoptar "una actitud colaborativa" (esuamp019). Por otra parte, tanto las actitudes pasivas (código 3.2) como las negativas (código 3.4), solamente se detectan entre los participantes de la UA que no han seguido un proceso de sensibilización.

\section{DISCUSIÓN}

Respecto a los resultados referidos a la Categoría 1. Aspectos de la carrera académica que limitan las posibilidades de tener hijas/os pasamos a interpretar los códigos más significativos. Esta categoría emerge en el contexto universitario de España, a diferencia del de otros países, debido a las nuevas problemáticas generadas por la implantación del EEES. Así, las titulaciones de Grado con una carga lectiva de 240 créditos ECTS, requieren una nueva cultura de trabajo por parte del discente: trabajo autónomo, trabajo en grupo, asistencia a tutorías, compromiso con el profesorado y con los compañeros, exposiciones, realización del Trabajo Fin de Grado, entre otros (Lebrero, 2007). Es por ello por lo que el alumnado aqueja una gran carga de trabajo que pueda autolimitar la maternidad/ paternidad.

El gasto económico derivado de los estudios, uno de los obstáculos descritos por los participantes, afecta al desarrollo de la maternidad/paternidad en la universidad. Este hallazgo coincide con el estudio realizado por Bermúdez et al., (2011) en el que se muestra que a pesar de que el número de matriculados en los estudios de doctorado son mayoritariamente mujeres, cuando no reciben becas para avanzar en sus tesis doctorales es imposible compatibilizar las mismas con sus compromisos familiares y, debido a ello, el rendimiento es menor que el de los hombres.

La planificación de horarios por parte de los centros universitarios (escuelas y facultades) en función de los espacios disponibles, los créditos ECTS y las demandas del profesorado; obvian las necesidades del alumnado. Aspecto que afecta especialmente a los estudiantes que desean compatibilizar los estudios con otras obligaciones, y que coincide con la respuesta más apoyada en el estudio desarrollado por Elboj Saso, Iñiguez Berrozpe y Valero Errazu (2013): “debido a que

Tabla IV. Frecuencias absolutas de la categoría 3

\begin{tabular}{lcccc}
\hline \multicolumn{1}{c}{ CÓDIGOS } & \%FA UV & FR UV & \%FA UA & FR UA \\
\hline 3.1 Actitud activa & $28,13 \%$ & 0,33 & $44,29 \%$ & 0,67 \\
\hline 3.2 Actitud pasiva & $0,00 \%$ & 0,00 & $2,86 \%$ & 0,04 \\
\hline 3.3 Actitud positiva & $56,25 \%$ & 0,67 & $38,57 \%$ & 0,59 \\
\hline 3.4 Actitud negativa & $0,00 \%$ & 0,00 & $1,43 \%$ & 0,02 \\
\hline 3.5 No define actitud & $15,63 \%$ & 0,19 & $12,86 \%$ & 0,20 \\
\hline Total & $100 \%$ & - & $100 \%$ & - \\
\hline
\end{tabular}


muchos de los alumnos y alumnas encuestados consideraban que las facilidades de horarios y distribución de las clases eran muy escasas, fundamentalmente para aquellos que debían compatibilizar sus estudios con un empleo o con obligaciones familiares" (p. 12).

Con referencia a la Categoría 2. Propuestas legislativas de mejora para la conciliación estudiantil-familiar hallamos diversos estudios que reflexionan sobre las mismas necesidades demandas por nuestros participantes. De este modo, las iniciativas universitarias diseñadas por Springer, Parker y Leviten-Reid (2009) para apoyar a los padres-estudiantes incluyen medidas específicas como: subsidios para el cuidado de niñas/os, recursos financieros para ayudar a contribuir a los honorarios de guardería del alumnado, licencia de paternidad remunerada (en un rango entre 2 y 12 semanas sin perder los privilegios de estar registrado como estudiante a tiempo completo), opciones de matrícula a tiempo parcial, cuidado infantil subsidiado y seguro médico/escolar. Los hallazgos de este estudio coinciden con las demandas de nuestros participantes respecto a: la necesidad de una baja de maternidad estudiantil, de un seguro escolar para lo hijos/as, así como las becas de estudio tanto para cubrir los gastos derivados de las responsabilidades académicas como para el cuidado infantil y el transporte. En este sentido, Brown y Nichols (2012) plantean propuestas específicas en el ámbito de la Educación Superior: ayudas financieras como préstamos con reducidos intereses, becas, donaciones, convenios de trabajo/estudio a tiempo parcial en el campus, o ayudantías en departamentos universitarios, y una política uniforme respecto a las condiciones de negociación de cara a la aceptación de las hijas e hijos de estudiantes en clases o tutorías. Esta última propuesta coincide con la demanda de nuestros participantes de asistir al aula con bebés.

A su vez Lynch (2008) reclama medidas relacionadas con las estructuras del entorno como el apoyo financiero a las madres y padres universitarios. En términos generales, los participantes de su estudio consideraron que se les ofrecía poco apoyo financiero y que las escasas ayudas recibidas no estaban pensadas para madres, sino más bien para personas sin hijas/os. La financiación institucional resultaba insuficiente para cubrir las necesidades inmediatas como la atención o escuela infantil, y la duración de los estudios universitarios. Lo que les obligaba a buscar fuera de la institución universitaria el apoyo financiero. El $80 \%$ de los entrevistados contemplaba los préstamos del gobierno (subvencionados y no subvencionados) como soluciones reales para aliviar las limitaciones financieras derivadas de combinar los estudios de grado y la maternidad/paternidad. En el contexto español no existe esta posibilidad de préstamo gubernamental y es por ello que ningún participante de nuestro estudio concibe esta alternativa.

Finalmente, la Categoría 3. Actitudes del alumnado adoptables a favor de la conciliación estudiantil-familiar recoge "la importancia del apoyo emocional y el aliento tácito y explicito, el respeto mutuo, la alabanza, el tiempo pasado con los tutores, el reconocimiento a la vida privada de los estudiantes y la creación de redes a favor de la madre estudiante" (Lynch, 2008, p. 599), como una oportunidad significativa para incrementar el capital humano (Shepherd y Mullins Nelson, 2012). Las madres y padres estudiantes precisan vincularse a las identidades socio-culturales a través de prácticas identitarias, y del apoyo del profesorado o asesoras/es, compañeras/os y familias (Lynch, 2008).

Los cuatro códigos que han emergido en esta categoría se podrían llegar a combinar de diversas formas como, por ejemplo: activa-positiva, activa-negativa, pasiva-positiva, pasiva-negativa. Una de estas combinaciones, la menos deseable, es precisamente la que visibiliza Bosch (2013) al referirse al sabotaje activo de algún compañero. El autor, además, plantea otras barreras para combinar estudios de postgrado y maternidad, manifestadas por las universitarias como: ser de una edad más avanzada y la falta de apoyo de los que les rodean. Para reducir esas actitudes discriminatorias son necesarias medidas como las propuestas por Springer, Parker y Leviten-Reid (2009) para crear una cultura universitaria adaptada: espacios familyfriendly, grupos de apoyo, celebración de actos sociales con familias a través de actividades que animen la asistencia de los menores, etc.

Demers (2014), centrada en aspectos relacionados con la salud de las madres universitarias y el estrés que padecen al tratar de equilibrar vida familiar, laboral y estudiantil, insiste en la importancia del apoyo de la familia, pero especialmente el apoyo de los iguales, de los compañeros de estudio. La compresión de la comunidad universitaria y su actitud es, por tanto, primordial para garantizar la conciliación estudiantilfamiliar, tal y como se demuestra nuestro estudio en la diferencia de resultados obtenidos entre los estudiantes de la Universidad de Alicante y los de la Universitat de València -que sí participaron en el proceso de sensibilización-. 


\section{CONCLUSIONES}

El conjunto de los relatos reflexivos nos ha permitido extraer las siguientes conclusiones. Aun siendo el colectivo de estudiantes madres minoritario, la imposibilidad de conciliar la maternidad con los estudios es una discriminación hacia las mujeres estudiantes respecto al resto del alumnado universitario. Por ello, las evidencias de esta investigación nos permiten concluir la necesidad de plantear mecanismos que fomenten la igualdad entre las madres universitarias y el resto del alumnado, para establecer no solo la oportunidad de estudiar, sino también, el derecho a la conciliación estudiantil-familiar. Asimismo, tras la revisión bibliográfica podemos afirmar que el término conciliación estudiantil-familiar no se halla en ninguna publicación científica, legislación, normativa o estadística nacional. La inexistencia del concepto pone de manifiesto que la idea es tan novedosa que ni siquiera la población en general o la comunidad científica ha comenzado a referirse a ella. Los hallazgos en este estudio nos permiten concluir la urgente necesidad de exponer esta problemática, ya que de lo contrario, mientras no se garantice legislativamente la conciliación estudiantil-familiar, la institución universitaria continuará reproduciendo procesos de discriminación hacia la mujer estudiante. La universidad es la institución donde se forman las futuras profesionales e investigadoras que crean conocimiento y ello permite que las sociedades avancen. Perpetuar estas limitaciones que afectan al colectivo de madres estudiantes, puede condicionar el desarrollo de la ciencia, aspecto muy destacado en la sociedades actuales.

A partir de las demandas de los participantes, consideramos necesarias propuestas de mejora encaminadas a reducir los aspectos de la carrera académica que limitan las posibilidades de tener hijas/os, tales como: la carga de trabajo académico, el gasto económico derivado de los estudios, o los horarios establecidos, entre otros. Aun siendo necesarias actuaciones desde el ámbito académico, apremia realizar cambios legislativos, en el seno de la institución universitaria, que permitan cuanto menos, garantizar derechos de maternidad/paternidad análogos a los que gozan las madres y padres trabajadores. La conciliación estudiantil-familiar no forma parte de la conciliación laboral-familiar, y, por tanto, no puede recogerse en la normativa interna de las universidades como una misma cosa. Precisa de regulaciones legislativas específicas porque las dificultades y necesidades del estudiante no coinciden con las del trabajador/a; y las dificultades y necesidades de la mujer estudiante no coinciden con las del hombre estudiante.
Por otra parte, consideramos que las voces de los participantes reclaman las siguientes acciones, que pueden favorecer la consecución de la conciliación estudiantil-familiar:

- Considerar el derecho a una "baja maternal de la mujer estudiante" -análoga a la baja a la que gozan las mujeres trabajadoras-.

- Adoptar medidas legislativas que favorezcan la conciliación estudiantil-familiar, tales como ayudas económicas; asistencia a clase con el bebé; y posibilidad de contratar un complemento al seguro escolar para hijas/os, en cualquier periodo del curso en el que se saben madres o padres.

El proceso de sensibilización respecto a la problemática de la falta de conciliación estudiantil-familiar, llevado a cabo con el alumnado de la UV, ha dado como resultado una visión más optimista, una mayor sensibilidad, capacidad reflexiva y constructiva en el colectivo. La comparación con el resto de los participantes de la UA, nos permiten determinar que si las instituciones universitarias no plantean acciones para la conciliación estudiantil-familiar, se perpetuarán los obstáculos y barreras para la maternidad/paternidad. Es preciso desarrollar campañas de sensibilización hacia la problemática de la conciliación estudiantil-familiar tanto en la comunidad universitaria (estudiantes, profesorado y personal administrativo y de servicios) como en la sociedad en general. Las necesarias campañas de sensibilización deben ir especialmente enfocadas a los sujetos que adoptan una actitud activa y a su vez negativa; pero también a aquellos que manifiestan una actitud pasiva-negativa o pasiva-positiva; y deberían aprovechar la predisposición de los sujetos activos y a la vez positivos.

Podemos actuar desde el ámbito universitario, en favor de la conciliación familiar-estudiantil, en dos sentidos: por un lado para paliar las dificultades por las que la maternidad/paternidad limitan el desarrollo académico (no objeto de este estudio); y por otro lado para suprimir, reducir, corregir o paliar las dificultades por las que la carrera académica pueda limitar las posibilidades de tener hijas/os (objeto de este estudio). Aunque en este estudio nos hemos centrado en las demandas de corte legislativo, la actuación para ambos frentes puede realizarse mediante propuestas académicas, legislativas o de implementación de recursos.

Sin la actuación global no es posible combatir el problema estructural de la discriminación de la mujer; en la limitación académica y en la limitación a la maternidad que afecta especialmente al género femenino. 
Los relatos que constituyen el corpus de esta investigación son abundantes y ricos, sin embargo, no podemos descuidar la posibilidad de ampliar la muestra ( $a$ otras titulaciones y a estudios posgrado) en futuras pesquisas. Asimismo, la ausencia de investigaciones sobre esta problemática, constatada por diversos investigadores (Brown y Nichols, 2012; Doble y Supriya, 2011; Shepherd y Mullins Nelson, 2012; Springer, Parker y Leviten-Reid, 2009), ha limitado poder contrastar algunas evidencias en los hallazgos descritos. No obstante, el estudio de la bibliografía que compone esta investigación justifica la problemática y la necesidad de plantear la misma como un nuevo campo para indagar en la Educación Superior.

\section{AGRADECIMIENTOS}

Queremos agradecer la colaboración de los estudiantes del Grado de Maestro de Educación Primaria de la Universidad de Alicante (UA) y de la Universitat de València (UV) que han participado de este estudio, gracias a los cuales ha sido posible la gestación de este manuscrito.

\section{NOTAS}

1. Dirigida solo a quienes tenían menos de 50 años y estaban embarazadas en ese momento: Cuando Ud. se quedó embarazada, ¿deseaba Ud. el embarazo, hubiera preferi-

\section{BIBLIOGRAFÍA}

Amorós, C. (1987). Espacio de los iguales, espacio de las idénticas. Notas sobre el poder y principio de individuación. Arbor, 11, pp. 113-127.

Amorós, C. (1990). Mujer: Participación, cultura política y Estado. Buenos Aires: Ediciones de la Flor.

Andreu, M. (2008). Los abandonos universitarios: retos ante el espacio europeo de educación superior. Estudios sobre Educación, 15, pp. 101-121.

Bermúdez, M. P., Guillén-Riquelme, A., Gómez-García, A., Quevedo-Blasco, R., Sierra, J. C. y Buela-Casal, G. (2011). Análisis del rendimiento en el doctorado en función del sexo. Educación XX1, 14, 1, pp. 17-33. http://dx.doi.org/10.5944/ educxx1.14.1.261

Bosch, B. (2013). Women who study: balancing the dual roles of postgraduate student and mother [Tesis doctoral inédita]. Edith Cowan University: Western Australia, Australia.

Brown, V. y Nichols, T. R. (2012). Pregnant and parenting students on campus: policy and program implications for a growing population. Educational Policy, 27, 3, pp. 499-530. http://dx.doi. org/10.1177/0895904812453995

Bugarleta, E. (2011). Género, identidad y consumo: las "nuevas maternidades" en España. [Tesis doctoral inédita]. Universidad Complutense de Madrid: Madrid.

Cabrera, L., Bethencourt, J., Álvarez, P. y González, M. (2006). El problema del

do esperar hasta más tarde o no quería quedarse embarazada bajo ningún concepto?

2. Consulta realizada por correo electrónico.

abandono de los estudios universitarios. RELIEVE, 12, 2, pp. 171-203. Disponible en: http://www.uv.es/RELIEVE/v12n2/ RELIEVEv12n2_1.htm

Camps, V. (1996). El siglo de las mujeres. Madrid: Cátedra.

Cerdá, M. R. (2009). Adenda. Plan de Igualdad en Universidad Pública: breve análisis líneas de actuación. En Jornadas Planes de Igualdad y Universidades. 22-23 de octubre de 2009. Santander: Universidad de Santander.

Clandinin, D. J. y Murphy, M. S. (2009). Comments on Coulter and Smith: Relational Ontological Commitments in $\mathrm{Na}$ rrative Research. Educational Researcher, 38, 8, pp. 598-692. http://dx.doi. org/10.3102/0013189X09353203

Corbin, J. y Strauss, A. (2008). Basics of qualitative research echniques and Procedures for Developing Grounded Theory (3a ed.). Thousand Oaks: Sage. http:// dx.doi.org/10.4135/9781452230153

Demers, D. (2014). Back to school: the balancing act graduate student mothers play between home and school [Tesis doctoral inédita]. Southern Illinois University Carbondale: Illinois, Estados Unidos.

Denzin, N. K. y Lincoln, Y. E. (eds.) (2011). Handbook of Qualitative Research (4a ed). Thousand Oak: Sage.

Doble, N. y Supriya, N. (2011). Student life balance: myth or reality? International Journal of Educational Manage- ment, 25, 3, pp. 237-251. http://dx.doi. org/10.1108/09513541111120088

Donoso, T., Figuera, P. y Rodríguez-Moreno, $M$. (2011). Barreras de género en el desarrollo profesional de la mujer universitaria. Revista de Educación, 355, pp. 187-212. http://dx.doi.org/10.4438/1988-592XRE-2011-355-021

Elboj Saso, C., Iñiguez Berrozpe, T. y Valero Errazu, D. (2013). Conciliación familiar y laboral también en las aulas del EEES. Una propuesta para prevenir el abandono de los estudios de Grado por motivos extraacadémicos. En Crisis y cambio: propuestas desde la sociología. XI Congreso español de sociología. Disponible en: http:// www.fes-web.org/uploads/files/modules/congress/11/papers/169.pdf

Escandell, M. O., Marrero, G., Castro, J. J. y Rodríguez, A. (2002). El abandono universitario: un estudio de la deserción en las titulaciones de Maestro y Educación Social. Anuario de Filosofía, Psicología y Sociología, 4, 5, pp. 81-83.

González Pérez, T. (2008). Las mujeres españolas en el sistema educativo. La construcción de programas y modelos formativos. Clepsydra, 7, pp. 77-92.

Huber, J., Caine, V., Huber, M. y Steeves, P. (2013). Narrative Inquiry as Pedagogy in Education: the extraordinary potential of living, telling, retelling, and reliving stories of experience. Review of Research in Education, 37, pp. 212-242. http://dx.doi. org/10.3102/0091732X12458885 
Huber, G. L. y Gürtler, L. (2013). AQUAD 7. Manual del programa para analizar datos cualitativos. Disponible en: http:// www.aquad.de/materials/manual_ aquad7/manual-c.pdf

Lebrero, M. P. (2007). Estudio comparado de los nuevos estudios de Grado de Educación Infantil en el Espacio Europeo de Educación Superior (EEES). Revista de Educación, 343, pp. 275-299.

López-Ibor, R. A., Escot, L. y Fernández, J. A. (2010). La predisposición de las estudiantes universitarias a auto-limitarse profesionalmente en el futuro por razones de conciliación. Estudios de Economía Aplicada, 28, 1, pp. 1-32.

Lorenzo, M. Argos, J. Hernández, J. y Vera, J. (2014). El acceso y la entrada del estudiante a la universidad: situación y propuestas de mejora facilitadoras del tránsito. Educación XX1, 17, 1, pp. 15-38. http://dx.doi.org/10.5944/educxx1.17.1.9951

Lynch, K. D. (2008). Gender roles and the American academe: a case study of graduate student mothers. Gender and Education, 20, 6, pp. 585-605. http://dx.doi. org/10.1080/09540250802213099

Maganto, J. M., Etxeberría, J. y Porcel, A. (2010). La corresponsabilidad entre los miembros de la familia, como factor de conciliación. Educatio Siglo $X X I, 28,1$, pp. 69-84. http://dx.doi. $\operatorname{org} / 10.6018 / \mathrm{j} / 109731$

Olesen, V. (2011). Feminist qualitative research in the millennium's first decade. Developments, challenges, prospects. En Denzin, N. K. y Lincoln, Y.E. (eds.) The SAGE Handbook of Qualitative Research (4a ed.). Thousand Oak: Sage, pp.129-146.

Saletti, L. (2008). Propuesta teórica feminista en relación al concepto de maternidad. Clepsydra, 7, pp. 169-183.

Shepherd, J. y Mullins Nelson, B. (2012). Balancing act: A phenomenological study of female adult learners who successfully persisted in graduate studies.
The Qualitative Report, 17 (art. 39), pp. 1-21. Disponible en: http://www.nova. edu/ssss/QR/QR17/shepherd.pdf

Springer, K. W., Parker, B. K. y LevitenReid, C. (2009). Making Space for Graduate Student Parents. Practice and Politics. Journal of Family Issues, 30, 4, pp. 435-457. http://dx.doi. org/10.1177/0192513X08329293

Tomàs Folch, M., y Mentado Labao, T. (2013). Las temáticas y preocupaciones de las investigadoras élite en Ciencias Sociales de las universidades catalanas. Arbor, 189, 760, a019. http://dx.doi. org/10.3989/arbor.2013.760n2005

Valcárcel, A. (1997). La política de las mujeres. Madrid: Cátedra.

\section{Textos legales}

Ley Orgánica 1/2004, de 28 de diciembre de Medidas de Protección Integral contra la Violencia de Género. Boletín Oficial del Estado del miércoles 29 de diciembre de 2004. Disponible en: https:// www.boe.es/boe/dias/2004/12/29/ pdfs/A42166-42197.pdf

Ley Orgánica 3/2007, de 22 de marzo, para la igualdad efectiva de mujeres y hombres. Boletín Oficial del Estado del viernes 23 de marzo de 2007. Disponible en: https://www.boe.es/boe/ dias/2007/03/23/pdfs/A12611-12645. pdf

Ley Orgánica 4/2007, de 12 de abril, por la que se modifica la Ley Orgánica 6/2001 de 21 de diciembre, de Universidades. Boletín Oficial del Estado del viernes 13 de abril de 2007. Disponible en: https:// www.boe.es/boe/dias/2007/04/13/ pdfs/A16241-16260.pdf

Real Decreto 1393/2007, de 29 de octubre, por el que se establece la ordenación de las enseñanzas universitarias oficiales. Boletín Oficial del Estado del martes 30 de octubre de 2007. Disponible en: http:// www.boe.es/boe/dias/2007/10/30/ pdfs/A44037-44048.pdf
Real Decreto 1791/2010, de 30 de diciembre, por el que se aprueba el Estatuto del estudiante universitario. Boletín Oficial del Estado del viernes 31 de diciembre de 2010. Disponible en: http:// www.boe.es/boe/dias/2010/12/31/ pdfs/BOE-A-2010-20147.pdf

\section{Recursos de Internet}

Centro de Investigaciones Sociológicas. Estudio no 2.639. Abril-mayo 2006. Fecundidad y valores en la España del siglo XXI. Avance de resultados. [En línea]. [Fecha de consulta: 25 de julio de 2014]. Disponible en: http://datos.cis.es/pdf/ Es2639mar_A.pdf

Datos básicos del sistema universitario español. Curso 2013-2014. [En línea]. [Fecha de consulta: 22 de julio de 2014]. Disponible en: http://www.mecd.gob. $\mathrm{es} / \mathrm{dms} / \mathrm{mecd} /$ educacion-mecd/areaseducacion/universidades/estadisticas-informes/datos-cifras/DATOS_ClFRAS_13_14.pdf

Encuesta de fecundidad 1999. [En línea]. [Fecha de consulta: 22 de julio de 2014]. Disponible en: http://ine.es/ jaxi/menu.do?type=pcaxis \&path=/t20/ p317\& file $=$ inebase $\& L=0$

INEbase: Operaciones estadísticas: clasificación por temas. [En línea]. [Fecha de consulta: 28 de noviembre de 2014]. Disponible en: http://www.ine.es/inebmenu/indice.htm

Recull de Dades Estadístiques. Curs 12-13. Universitat de València. Servei d'Anàlisi i Planificació. [En línea] [Fecha de consulta: 24 de julio de 2014] Disponible en: http://www.uv.es/sap/docs/reculls/ recull1213.pdf 